\title{
Nachhaltigkeit in der
}

\section{Sozialen Arbeit}

\section{Konzeptionelle, praktische und empirische Implikationen aus pädagogischer Sicht am Beispiel der Jugendsozialarbeit}

\section{JOACHIM KÖNIG}

Prof. Dr. Joachim König ist Hochschullehrer für Allgemeine Pädagogik und Empirische Sozialforschung an der Evangelischen Hochschule Nürnberg. Er ist gleichzeitig Leiter des Instituts für Praxisforschung und Evaluation an dieser Hochschule.

E-Mail joachim.koenig@evhn.de

\author{
Das Konzept der Nachhaltigkeit beruht darauf, \\ Ressourcen nur so stark zu nutzen, dass sie auf \\ natürliche Weise regeneriert werden können. \\ Dieses Verständnis kann auch auf die Soziale \\ Arbeit angewendet werden, wie am Beispiel der \\ Jugendsozialarbeit gezeigt werden kann.
}

Dieser Beitrag versucht, den in der letzten Zeit sehr inflationär und gleichzeitig recht unscharf verwendeten Begriff der Nachhaltigkeit zunächst allgemein und dann aus der Sicht der Pädagogik zu bestimmen und zu entfalten. (1)

Vor diesem Hintergrund wird ein Vorschlag unterbreitet, wie er für die Soziale Arbeit - am Beispiel der Jugendsozialarbeit - in konzeptioneller, praktischer und empirischer Hinsicht fruchtbar gemacht werden kann. Dazu werden drei Bezugsebenen entfaltet:

- der individuelle Kern des Begriffs, orientiert am Menschenbild einer Person mit unverfügbaren Rechten und mit sich aus ihnen ergebenden Pflichten

- die gesellschaftliche Ebene, orientiert am Postulat der Gerechtigkeit und an den Konsequenzen, die sich für eine mündige Person in ihrer Lebenswelt daraus ergeben

- der zeitliche Horizont, orientiert an einem Verständnis von Entwicklung, das die rein formale Betrachtung von Veränderungen in qualitativer, den Sinn von Veränderungen berücksichtigender Hinsicht überwindet.

Vor dem Hintergrund dieser drei Ebenen werden darauf aufbauend Ansätze zur Umsetzung in eine nachhaltige Praxis und zur Operationalisierung für eine Empirie der Nachhaltigkeit angedeutet.

\section{Was Nachhaltigkeit bedeutet}

Geradezu inflationär hat sich der Gebrauch der Vokabel "Nachhaltigkeit « in den letzten Jahren entwickelt. Und doch feiert sie im letzten Jahr bereits ihren dreihundertsten Geburtstag: Nachdem nämlich Hans Carl von Carlowitz 1711 zum Oberberghauptmann am kursächsischen Hof in Freiberg in Sachsen ernannt worden ist, erkannte er - zuständig für die dortige Forstwirtschaft als Zulieferer für den Bergbau - das damals schon ökonomische und zugleich ökologische Problem sofort: Der ständig steigende Holzbedarf führte zu Abholzungen, die den Waldbestand in Sachsen in seiner Existenz bedrohte. So schrieb er in seiner »Sylvicultura Oeconomica « mit dem schönen Untertitel »Anweisung zur Wilden Baumzucht« (1): »... wird derhalben die größte Kunst, Fleiß und Einrichtung hiesiger Lande darinnen beruhen, wie eine sothane Conservation und Anbau des Holtzes anzustellen, $\mathrm{da} ß$ es eine continuierliche beständige und nachhaltende Nutzung gebe, weiln es eine unentberliche Sache ist, ohne welche das Land in seinem Esse nicht bleiben mag. «Dies war - wenn wir den Quellen glauben dürfen - die Geburtsstunde des Grundgedankens der Nachhaltigkeit im Sinne der Sorge um die langfristige Erhaltung der Grundlagen für die natürliche und wirtschaftliche Existenz der Menschen.

Danach wurde immer wieder dieser Grundgedanke des zukunftsverant- 
Matrix zu methodischen und empirischen Umsetzung des Prinzips der Nachhaltigkeit in der Praxis

\begin{tabular}{|c|c|c|c|c|}
\hline \multirow{2}{*}{$\begin{array}{l}\text { Prinzipien } \\
\text { der Nach- } \\
\text { haltigkeit }\end{array}$} & \multirow{2}{*}{$\begin{array}{l}\text { Beispiel aus } \\
\text { dem Bereich: }\end{array}$} & \multirow[t]{2}{*}{ Ziele } & \multicolumn{2}{|c|}{ Umsetzung } \\
\hline & & & $\begin{array}{l}\text { Konzeptionell - praktisch handelnd: } \\
\text { Inhalte \& Methoden }\end{array}$ & $\begin{array}{l}\text { Evaluativ - empirisch bewertend: } \\
\text { Indikatoren \& Methoden }\end{array}$ \\
\hline $\begin{array}{l}\text { Mündige } \\
\text { Person }\end{array}$ & $\begin{array}{l}\text { Arbeitswelt- } \\
\text { bezogene } \\
\text { Jugendsozial- } \\
\text { arbeit (1) }\end{array}$ & $\begin{array}{l}\text { Erlernen von Strategien zur } \\
\text { Gewinnung von Mündigkeit } \\
\text { und Teilhabe. } \\
\text { Verbesserung der kommunalen } \\
\text { Finanzierung von } \\
\text { Jugendsozialarbeit. }\end{array}$ & $\begin{array}{l}\text { Teilnahme an Stadtratssitzungen zur } \\
\text { kommunalen Finanzierung von } \\
\text { Jugendsozialarbeit. } \\
\text { Offene Briefe, Plakate. } \\
\text { Einladung von PolitikerInnen in die } \\
\text { Einrichtung mit Fragestunde. }\end{array}$ & $\begin{array}{l}\text { Dokumentation positiver Rückmeldungen. } \\
\text { Interviews zur Veränderung des politischen } \\
\text { Bewusstseins bei den Jugendlichen. } \\
\text { Fragebögen zur Zufriedenheit und zur } \\
\text { gefühlten Integration in die Gesellschaft. }\end{array}$ \\
\hline $\begin{array}{l}\text { Gerechte } \\
\text { Lebenswelt }\end{array}$ & $\begin{array}{l}\text { Jugendsozial- } \\
\text { arbeit an } \\
\text { Schulen (2) }\end{array}$ & $\begin{array}{l}\text { Familien in die Beratung mit } \\
\text { einbeziehen und in die Schule } \\
\text { integrieren. } \\
\text { Verbesserung der } \\
\text { Zugangsgerechtigkeit zu } \\
\text { Beratung und Hilfen. } \\
\text { Stärkung der Mitarbeit und der } \\
\text { Übernahme von } \\
\text { Verantwortung bei den Eltern. }\end{array}$ & $\begin{array}{l}\text { Offensive Werbung für zusätzliche, } \\
\text { niedrigschwellig angelegte Beratungstage } \\
\text { für Eltern von MigrantInnen. } \\
\text { Gestaltung von kulturellen Events, } \\
\text { Schulfesten und Feiern. }\end{array}$ & $\begin{array}{l}\text { Dokumentation der Inanspruchnahme von } \\
\text { Beratungsstunden für die Eltern. } \\
\text { Interviews mit den Eltern im Hinblick auf } \\
\text { die Wirkung ihrer Beteiligung in den } \\
\text { Familien. } \\
\text { Fragebögen zur Verbesserung der } \\
\text { Beteiligung der Eltern am schulischen und } \\
\text { öffentlichen Leben. }\end{array}$ \\
\hline $\begin{array}{l}\text { Sinnvolle } \\
\text { Entwick- } \\
\text { lung }\end{array}$ & $\begin{array}{l}\text { Gender- } \\
\text { orientierte } \\
\text { Jugendsozial- } \\
\text { arbeit mit } \\
\text { MigrantInnen } \\
\text { (3) }\end{array}$ & $\begin{array}{l}\text { Integration als einen } \\
\text { langfristigen, individuell } \\
\text { sinnvollen } \\
\text { Entwicklungsprozess } \\
\text { verstehen. }\end{array}$ & $\begin{array}{l}\text { Biographisches Arbeiten mit Mädchen im } \\
\text { Rahmen des Nachmittagsprogramms an } \\
\text { Ganztagesschulen. }\end{array}$ & $\begin{array}{l}\text { Dokumentation des Beteiligungsverhalten } \\
\text { der Mädchen anhand einer } \\
\text { Beobachtungsmethode. } \\
\text { Fragebögen zur gesellschaftlichen } \\
\text { Integration der Mädchen und ihrer Familien } \\
\text { mit geschlossenen und offenen Fragen. }\end{array}$ \\
\hline
\end{tabular}

wortlichen Denkens und Handelns im Sinne des Wohles des Menschheit auch für künftige Generationen in seiner Bedeutung hervorgehoben und schließlich in einem »Drei-Säulen-Modell der Nachhaltigkeit« zusammengefasst und von Rademacher (3) hervorragend erläutert. Nachhaltigkeit bezeichnet demnach eine dauerhaft zukunftsfähige Entwicklung der ökonomischen, ökologischen und sozialen Dimension einer Gesellschaft:

- Ökologisch nachhaltig ist eine Lebensweise, die alle natürlichen Ressourcen nur in einem Umfang beansprucht, wie sie sich regenerieren. Natur und Umwelt werden für das Leben der nachfolgenden Generationen erhalten.

- Ökonomisch nachhaltig ist Wirtschaft, wenn langfristige Planungshorizonte die Basis der unternehmerischen Entscheidungen sind, die Belange der Umwelt und der Gesellschaft mit in das ökonomische Denken einbezogen werden und insbesondere ein Subsistenz garantierender Erwerb aller Mitglieder einer Gesellschaft ermöglicht wird.

- Soziale und gesellschaftliche Nachhaltigkeit ist gegeben, wenn die In- teressen unterschiedlicher sozialer Gruppen berücksichtigt und zukunftsverantwortlich im Sinne einer lebenswerten Gemeinschaft gefördert werden.

Soziale Nachhaltigkeit als eine dieser drei Säulen ist inzwischen aus volkswirtschaftlicher und sozialpolitischer sowie aus ökologischer Sicht intensiv diskutiert worden. Inzwischen sind es im Wesentlichen fünf Leitorientierungen, die als Grundlage für eine begriffliche Bestimmung sozialer Nachhaltigkeit mit Blick auf die in einer Gesellschaft zusammenlebenden Individuen gelten können (4):

- Eine menschenwürdige Existenz aller im Sinne ihrer materiellen und immateriellen Grundbedürfnisse kann als die zentrale Grundforderung angesehen werden.

- Eine Existenzsicherung durch gerechte Arbeit in einem ganzheitlichen Sinne (also nicht nur auf die klassische Erwerbsarbeit bezogen) muss dazu ermöglicht werden können.

- Soziale Ressourcen für Handlungschancen und Handlungsperspektiven müssen zur Verfügung stehen.

- Chancengleichheit muss auch in einer Gesellschaft, die durch zunehmende soziale Ungleichheit gekennzeichnet ist, trotz allem als Maxime aufrechterhalten werden.

- Beteiligungsmöglichkeiten für alle Gruppen zu schaffen und im Sinne von Niedrigschwelligkeit diese zugänglich zu machen, bleibt eine sozialpolitische Aufgabe.
"Nachhaltigkeit hat drei

Dimensionen: eine ökologische, eine ökonomische und eine soziale» 


\section{Was Nachhaltigkeit in der Sozialen Arbeit bedeuten kann}

Aus originär sozialwissenschaftlicher, dabei vor allem aus pädagogischer Perspektive, gibt es bisher kaum Konzeptionsversuche, obwohl die Forderung nach Nachhaltigkeit in den Feldern der Sozialen Arbeit inzwischen Eingang in die Begrifflichkeiten, Konzepte und Rhetoriken gefunden hat.

Aus allgemeinpädagogischer Sicht finden sich mindestens drei Verbindungslinien für eine begriffliche Bestimmung des Konzeptes der Nachhaltigkeit und sinnvolle Möglichkeiten zur Operationalisierung des Begriffs im praktischen, konzeptionellen und empirischen Zusammenhang, aus denen heraus Kriterien zur Beschreibung und Bewertung von Praxis formuliert werden können:

- Mündigkeit als Verantwortung einer Person definieren: Das zentrale Ziel aller Erziehungs- und Bildungsbemühungen ist Mündigkeit. (5) Ausgangspunkt ist der Grundgedanke, dass Verantwortung im Sinne von Mündigkeit immer mit den Rechten und mit den Pflichten einer Person untrennbar verbunden ist. Beides ist wiederum sowohl an die Person selbst als auch auf ihre Lebenswelt und die sie umgebende Gesellschaft gerichtet. Die »Medaille der Mündigkeit" hat also sowohl eine individuelle als auch eine sozial-gesellschaftliche Seite: Neben dem Recht auf Selbstbestimmung wird einer mündigen Person immer auch die Pflicht zur Selbstverantwortung auferlegt, neben ihrem Recht auf Mitbestimmung muss immer auch die Pflicht zur Mitverantwortung gesehen und eingefordert werden. Insofern geht der Begriff der Person in seiner pädagogischen Bedeutung ganz wesentlich über das hinaus, was in eher neutraler Form soziologisch mit dem Begriff des Individuums gemeint ist.

- Bildung als gerechte Teilhabe und Partizipation begreifen: Grundlegende Voraussetzung und Basis für alle Bildungsprozesse ist die individuelle Erfahrung, das Dazulernen im elementaren Sinne. Wissen zu erwerben, um die Welt kennenzulernen, um Zusammenhänge, Strukturen und Abläufe zu verstehen, ist notwendige Grundlage für alle weiteren »Bildungsschritte «, bei denen es dann darum gehen kann, dieses erworbene Wissen auch anzuwenden, also in einem zweiten Schritt Kompetenzen zu erwerben im Umgang mit Wissen und sich Strategien anzueignen, eigene Lernprozesse künftig auch selbst steuern zu können, also auch zu lernen, wie man lernt. Auf einer dritten Stufe muss es dann darum gehen, Bildung als einen Prozess der Entwicklung einer eigenen Ethik zu verstehen und umzusetzen: Sich vor dem Hintergrund des erworbenen Wissens eine eigene Meinung, einen bewertenden Standpunkt zu »bilden «, begründetes Urteilen zu lernen. So kann sukzessive - auf einer vierten Stufe angesiedelt, ein Bewusstsein entstehen, das es von dieser Grundlage aus erlaubt, auch handelnd in einem Gemeinwesen einzugreifen, also dann auch gezielt Einfluss zu nehmen und so Gesellschaft und Lebenswelt mit zu gestalten, auf

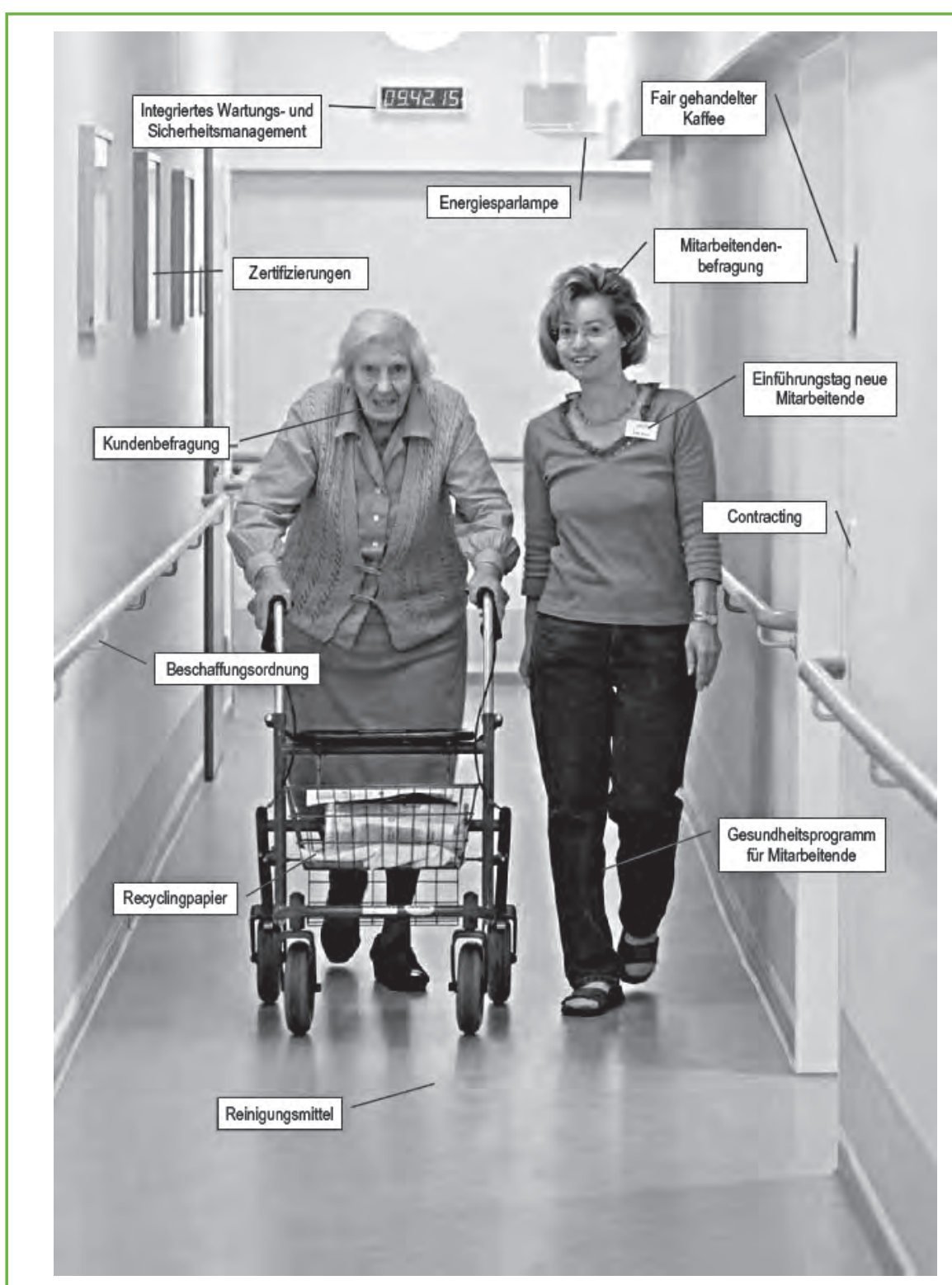

Die "Dienste für Menschen « mit Sitz in Stuttgart betreibt als großer diakonischer Altenhilfeträger Pflegestifte, Wohnstifte, ambulante Dienste, eine Geriatrische Rehabilitationseinrichtung sowie ein Geriatrisches Fachkrankenhaus. Ein besonderes Gewicht legt die Organisation auf nachhaltiges Wirtschaften. Dazu dient ein eigens entwickeltes »Nachhaltigkeitsmanagement-System EMASplus " sowie ein »Nachhaltigkeitsbericht«, aus dem die oben stehende Abbildung stammt.

Dienste für Menschen gGmbH, Haußmannstraße 103 A, 70188 Stuttgart, Telefon 0711 26879-0, E-Mail Kontakt@udfm.de, Internet http://www.udfm.de 
die Bedingungen des Zusammenlebens von Menschen mitzugestalten. Partizipation als die konsequente Weiterführung der bisherigen Stufen könnte so als immanenter Bestandteil und ein zentrales Ziel von Bildungsprozessen im Sinne der Mündigkeit einer Person beschrieben werden.

- Langfristig denken und sinnvolle Kontinuität wahren: Über das Tagesgeschäft hinaus denken, die Tragfähigkeit von Bemühungen für künftige Entwicklungen sichern, dies sind dem Begriff der Nachhaltigkeit immanente Forderungen, die pädagogischen Grundvorstellungen direkt entsprechen. Erziehung und Bildung als die beiden zentralen Kategorien in diesem Zusammenhang sind - etwa im Gegensatz zu den Begriffen des Lernens und der Qualifizierung - auf zunehmende Langfristigkeit und intrinsische Motivation im Sinne von Selbstständigkeit und Selbstverantwortlichkeit angelegt. Die Forderung nach lebenslangem Lernen hat in diesem Zusammenhang einen intensiven Einfluss auf die Bildungsdebatte über viele Jahre hinweg gehabt.

Aus den so angedeuteten Verbindungslinien können drei Konsequenzen für die Praxis, für das Alltagsgeschäft, etwa in der Jugendsozialarbeit, gezogen werden. Pädagogisches Denken und Handeln, das in der Sozialen Arbeit auf Erziehung und Bildung abzielt und an Nachhaltigkeit orientiert ist, muss drei Bedingungen erfüllen:

- ganzheitlich, also die Verantwortung des Individuums als Person ernst nehmend

- lebensweltlich, also die Umwelt und die gerechte Teilhabe in ihr mit einbeziehend und

- langfristig, also sinnvoll über das Tagesgeschäft hinaus.

Diese drei Bedingungen ernst zu nehmen, könnte bedeuten, aus ihnen für die Praxis der Sozialen Arbeit »Prinzipien der Nachhaltigkeit « abzuleiten, die für ihren konzeptionellen Zusammenhang sowie für die empirischen Fragen nach der Bewertung ihrer Praxis Bedeutung gewinnen könnten:

- Personen als Ganzes ernst nehmen, anstatt nur als »Fälle« zu betrachten:
Da geht es um das Menschenbild, das wir in der Alltagspraxis mit uns herumtragen: Die Person sollten wir in diesem Sinne als Ganzes betrachten. Damit ist gemeint, dass wir es mit mehr zu tun haben als mit einem Fall, einem Defizit, einem Symptom oder einem Delikt und dass wir von den drei Grundannahmen ausgehen, dass erstens jeder Mensch eine unteilbare Würde und unverfügbare Rechte besitzt, zweitens ein Individuum grundsätzlich aus sich heraus, zu subjektiv sinnvollem Handeln fähig ist und drittens allen Menschen die Fähigkeit zur Selbst- und Mitbestimmung und damit zur Selbst- und Mitverantwortung, die wir Mündigkeit nennen, zugetraut werden kann - und immer wieder einfordert werden muss.

- Nicht nur Individuen betrachten, sondern an gerechten Lebenswelten arbeiten: Da geht es vor allem um den systemischen Blick. Also um die Fähigkeit, im Alltagsgeschäft beispielsweise der Jugendsozialarbeit mit den Jugendlichen über das "Zweierverhältnis« - mein Klient und ich - hinauszudenken und den Sozialraum mit zu bedenken und in die Arbeit einzubeziehen, also diesen berühmten Grundsatz der Lebensweltorientierung und der Ressourcenorientierung, den Hans Thiersch (6) formuliert hat, ernst zu nehmen. Außerdem erscheint es notwendig, dass wir dabei auf die Niedrigschwelligkeit der Angebote achten und gleichzeitig auch das Prinzip der Stärkenorientierung insofern ernst nehmen, als wir von den Jugendlichen die Mitverantwortlichkeit für das Gemeinsame einfordern können und sollen.

- Sinnvolle Entwicklungen anstreben, statt nur auf kurzfristige Veränderungen setzen: An dem Punkt sollten wir uns fragen, was für ein Verständnis von Langfristigkeit und Entwicklung wir im pädagogischen Geschäft denn haben. Wichtig wäre da, dass wir über einzelne Veränderungen in einzelnen Situationen hinausdenken und Entwicklung als etwas Ganzheitlicheres begreifen, das mit der positiven Bewertung einer Veränderung verbunden sein muss.

\section{Was Nachhaltigkeit in der Jugendsozialarbeit bedeuten könnte}

Im Folgenden sollen nun anhand von drei Beispielen aus der Jugendsozialarbeit die Möglichkeiten angedeutet werden, wie sich nachhaltige Soziale Arbeit konzeptionell-praktisch und evaluierend-empirisch darstellen kann.

- Arbeitsweltbezogene Jugendsozialarbeit: Im Rahmen der »Bildungstage« einer Jugendwerkstätte, in der Jugendliche in Maßnahmen nach SGB II und SGB VIII sozialpädagogisch begleitet und beruflich qualifiziert werden, steht das Thema politisches Engagement auf der Agenda. Den Jugendlichen wird vermittelt, wie die Finanzierung der Maßnahmen funktioniert und es entwickelt sich eine Debatte darüber, wie denn Formen der Beteiligung und der Artikulierung der Interessen von Jugendlichen in diesem Zusammenhang aussehen könnten. Am Ende des Tages, an dem in Arbeitsgruppen verschiedene Aktionen entwickelt und geplant werden, wird entschieden, dass in den kommenden Wochen erstens ein gemeinsamer Besuch einer Sitzung des Jugendhilfeausschusses und anschließend des Stadtrates, die sich beide mit der Finanzierung von Jugendsozialarbeit befassen, stattfinden soll; zweitens eine Plakataktion zur Förderung des öffentlichen Bewusstseins über die Situation von benachteiligten Jugendlichen auf dem Arbeitsmarkt im Stadtteil gestartet werden soll und drittens für den nächsten Tag der offenen Tür in der Einrichtung Politiker zu einer Diskussions- und Fragerunde gebeten werden, bei der die Jugendlichen die Möglichkeit haben, auf ihre Situation hinzuweisen, Fragen zu stellen und auch ihre Forderungen an die Politik zu Ausdruck bringen können. Sowohl mit Blick auf die Dokumentation dieser Aktionen als auch mit dem Ziel ihrer Bewertung planen die Fachkräfte im Anschluss die Abfassung eines Berichts, der positive und kritische Rückmeldungen aus der Öffentlichkeit enthält, den Inhalt leitfadengestützter Interviews mit Jugendlichen im Hinblick auf die Wirkungen dieser Aktionen 
darstellt und die Ergebnisse einer kleinen Fragebogenuntersuchung systematisch dokumentiert, bei der alle Jugendlichen nach ihrer Zufriedenheit mit dem Erfolg der Maßnahmen und nach den Veränderungen der von ihnen wahrgenommenen Zugehörigkeit zur Gesellschaft gefragt werden. Diese Dokumentation wiederum wird genutzt als Teil einer größeren Kampagne zur Bedeutung der Jugendsozialarbeit.

- Jugendsozialarbeit an Schulen: Im Rahmen einer neu geschaffenen Stelle für Jugendsozialarbeit an einer städtisch geprägten Hauptschule initiiert die Sozialpädagogin eine neue Form der Elternarbeit. Das Ziel: Zum einen soll die Begleitung und Unterstützung der Jugendlichen auf eine breitere und ganzheitlichere Basis gestellt und zum anderen soll ein Beitrag zu Verbesserung der Integration der Eltern mit Migrationshintergrund geleistet werden. Dazu werden zusätzliche Beratungstage für Eltern eingeführt und »Kulturevents « an der Schule veranstaltet, mit einer ausdrücklichen Einladung an die Eltern und mit der Möglichkeit ihrer Beteiligung über Darbietungen oder kulinarische Beiträge. Im Rahmen der vom Sozialministerium als Finanzier der Stelle geforderten Evaluation werden diese zusätzlichen Maßnahmen einer empirischen Bewertung unterzogen. Die Sozialpädagogin dokumentiert die Häufigkeiten der Inanspruchnahme ihrer Beratungsstunden, führt eine Fragebogenerhebung jeweils nach den Kulturevents bei der Schülern und bei den Eltern durch (Zufriedenheit, Verbesserungsvorschläge, Verbesserung von Integration bei Migranten) und bittet einige Eltern darum, in halbstrukturierten Interviews Auskunft darüber zu geben, wie Sie die Arbeit an der Schule erleben, vor allem bezogen auf die Fragen nach Zugangsgerechtigkeit und nach den Integrationspotenzialen in ihrer Lebenswelt im Stadtteil. Im Rahmen einer externen Gesamtevaluation aller zusätzlichen Stellen für Jugendsozialarbeit an Schulen wird dann eine Metaanalyse erstellt, wie sich solche Effekte für ein Bundesland insgesamt sozialpolitisch darstellen und welche Wirkungen sich im Hinblick auf die soziale und berufliche Integration der Schülerinnen und Schüler daraus ergeben.

- Genderorientierte Jugendsozialarbeit mit Migrantinnen und Migranten: Im Rahmen der Entwicklung einer die Schulpädagogik unterstützenden Konzeption von Nachmittagsbetreuung bei der Einführung der Ganztagesschule entstehen neue Angebote für verschiedene Zielgruppen, u. a. für Mädchen der 8. bis 10. Jahrgangsstufe, die einen Migrationshintergrund aufweisen und zudem als eher sozial benachteiligt gelten. Biografiearbeit als eine inzwischen sozialpädagogisch gut entwickelte Methode wird für diese Mädchen in kleinen Gruppen angeboten. Inhaltlich versucht die Sozialpädagogin dabei, sich mit den Mädchen über deren Herkunft und ihre bisherigen Erfahrungen auszutauschen, um vor diesem Hintergrund Lebensziele, früher wie heute bewusster $\mathrm{zu}$ machen und darauf aufbauend Strategien entwickeln zu können, eigene Ziele systematischer und bewusster verfolgen und die Entwicklung der eigenen Persönlichkeit positiver beeinflussen zu können. Nicht nur eine Verbesserung der Integration der Mädchen erhoffen sich die beteiligten Akteure dadurch, sondern auch, einen Beitrag zur Steigerung des Selbstbewusstseins und des Selbstwertgefühls der Mädchen leisten zu können. Weil es sich bei diesem Vorhaben um ein Projekt mit Pilotcharakter handelt, soll es mit einer kleinen Selbstevaluation bewertet werden, um aus den Ergebnissen mögliche Konsequenzen für ähnliche Maßnahmen ableiten zu können. Dazu dokumentiert die Sozialpädagogin das Beteiligungsverhalten der Mädchen anhand eines Beobachtungsleitfadens und bittet die Mädchen, einen Fragebögen in anonymer Form zu bearbeiten, in dem sie nach ihren Erfahrungen mit der Biografiearbeit und nach den erlebten Veränderungen dadurch ein halbes Jahr später befragt werden, auch im Hinblick auf Entwicklungen, die zur Verbesserung der Integration dieser Zielgruppe beigetragen haben könnten.

\section{Fazit}

Nehmen wir den Anspruch auf Nachhaltigkeit für die Soziale Arbeit ernst, hier insbesondere und beispielhaft für die Jugendsozialarbeit, so könnte sich insgesamt betrachtet ein Verständnis von Nachhaltigkeit entwickeln, das sich an drei Prinzipien konzeptionell sowie praktisch orientiert und - je Arbeitsfeld spezifisch operationalisiert - auch messen lässt:

Erstes Prinzip: Jugendliche sollen ernst genommen und als mündige Personen in das Zentrum der Bemühungen gerückt werden. Nachhaltige Soziale Arbeit orientiert sich an einem christlich-humanistischen Menschenbild, das eine Person als Subjekt mit unverfügbaren Rechten und einer unveräußerlichen Würde versteht und ihm einen individuellen »Eigensinn« zugesteht, der Grundlage für alle Formen der Hilfe, Unterstützung, Beratung und Bildung ist. Andererseits wird einer Person neben ihrem sich daraus ergebenden Recht auf Selbstbestimmung immer auch die Pflicht zur Selbstverantwortung auferlegt und eingefordert.

Zweites Prinzip: Auf den verschiedenen gesellschaftlichen Ebenen sollen Beiträge zu einer gerechten Lebenswelt für die Jugendlichen geleistet werden. Nachhaltige Soziale Arbeit geht vor dem Hintergrund der ersten Prämisse grundsätzlich von der Mündigkeit ihrer Klientinnen und Klienten aus. Sie orientiert sich deshalb konsequent an den Ressourcen einer Person. Sie bezieht daher die Lebenswelten in alle Hilfen mit ein und formuliert vor diesem Hintergrund neben dem Recht auf Mitbestimmung einer Person auch ihre Pflicht zur Mitverantwortung. Gerechtigkeit als zentraler Maßstab muss dabei immer wieder zum Ziel der Interventionen in der Lebenswelt der Jugendlichen und zusammen mit den Jugendlichen werden.

Drittes Prinzip: Der individuelle Sinn des zeitlichen Horizonts hat entscheidende Bedeutung und soll deshalb zum Maßstab für die Qualität von Entwicklung werden. Nachhaltige Soziale Arbeit orientiert sich an einem Verständnis von Entwicklung, das über die Betrachtung von bloßen Veränderungen hinausgeht und dabei sowohl die Langfristigkeit als auch die Sinnhaftigkeit von Veränderungen aus einer individuellen (die Person 
betreffenden) und gemeinschaftlichen (die Lebenswelt betreffend) Perspektive und Verantwortung herausfordert. Ein solches Verständnis von Entwicklung wird dabei umso mehr zum zentralen Maßstab für Nachhaltigkeit, je besser der Anspruch der Partizipation, also der Fähigkeit und der Bereitschaft der Jugendlichen selbst, sich aktiv für eine gelingende Entwicklung in ihrem Leben und in der Gesellschaft einzusetzen, verwirklicht werden kann.

\section{Anmerkungen}

(1) Der Beitrag ist unter anderem vor dem Hintergrund eines Workshops der Bundesarbeitsgemeinschaft Evangelische Jugendsozialarbeit und der Evangelischen Jugendsozialarbeit in Bayern zu diesem Thema entstanden. Der Autor dankt allen Teilnehmenden für wertvolle Anregungen und die engagierte Diskussion.

(2) Carlowitz, H. C. (1713), S. 105 f.

(3) Vgl. Rademacher, F.-J. (2007).
(4) Vgl. dazu Embacher \& Wehling (2002), S. $32 \mathrm{ff}$.

(5) Vgl. dazu z. B. die ursprünglichen Überlegungen bei Kant (1799).

(6) Vgl. Thiersch, H. (2005).

\section{Literatur}

Carlowitz, H. C. (1713). Sylvicultura oeconomica. Anweisung zur wilden Baum. Leipzig: Braun.

Diefenbacher. H. (2001). Gerechtigkeit und

Nachhaltigkeit. Zum Verhältnis von Ethik und Ökonomie. Darmstadt.

Embacher, C. \& Wehling, P. (2002). Soziale

Dimensionen der Nachhaltigkeit. Theoretische

Grundlagen und Indikatoren. Studientexte.

Frankfurt am Main: Institut für sozial-ökologische Forschung.

Kant, I. (1799). Beantwortung der Frage: Was ist Aufklärung. In: J. H. Tieftrunk (Hg.), Immanuel Kants vermischte Schriften, Bd. 2. Halle.

McKenzie, S. (2004). Social Sustainability:

Towards Some Definitions. Magill: Hawk Research Institute. Working Paper Series No. 27.

Meadows, D. L. et al. (1972). The Limits To Growth. Universe Books.
Mittnacht, B. (2010). Qualitätsentwicklung und Nachhaltigkeit im Kontext häuslicher Pflegearrangements. Entwicklungstrends und Perspektiven. Lage: Jakobs-Verlag.

Oerter, M. \& Montada, L. (2008). Entwicklungspsychologie. Ein Lehrbuch. Weinheim: Beltz, PVU, 2008

Radermacher, F.-J. \& Beyers, B. (2007). Welt mit Zukunft. Überleben im 21. Jahrhundert. Hamburg: Murmann.

Renn, 0. (2007). Leitbild Nachhaltigkeit. Eine normativ-funktionale Konzeption und ihre Umsetzung. Wiesbaden: Verlag für Sozialwissenschaften

Ritt, Th. (2002). Soziale Nachhaltigkeit: Von der Umweltpolitik zur sozialen Nachhaltigkeit. Informationen zur Umweltpolitik, 149. Wien: Bundeskammer für Arbeiter und Angestellte. Spangenberg, J.H. (2002). Soziale Nachhaltig keit. Eine integrierte Perspektive für Deutschland. In: A. Dally \& B. Heins (Hg.), Politische Strategien für soziale Nachhaltigkeit. Loccum. S. $23-38$.

Thiersch, H. (2005). 6. Auflage. Lebensweltorientierte Soziale Arbeit. Aufgaben der Praxis im sozialen Wandel. Weinheim, München: Juventa.

\section{Das neue Beraterhandbuch.}

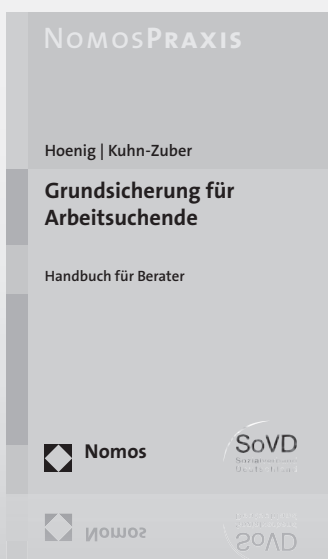

Das Werk berücksichtigt zum frühest möglichen Zeitpunkt sämtliche

Neuerungen und

- erläutert die konkreten Auswirkungen der Neuregelungen im Bereich SGB II wie III,

- berücksichtigt die Schnittmengen mit anderen Rechtsgebieten, wie z.B. dem Pflegerecht, dem Krankenversicherungsrecht oder dem Schwerbehindertenrecht und

- integriert eine Vielzahl von Beispielen und Hinweisen aus der alltäglichen Beratungspraxis.

\section{Grundsicherung für Arbeitsuchende}

Handbuch für Berater

Von Ragnar Hoenig und Prof. Dr. Gabriele Kuhn-Zuber

2012, ca. 350 S., brosch., ca. 49,- $€$,

ISBN 978-3-8329-6770-3

Erscheint ca. Mai 2012

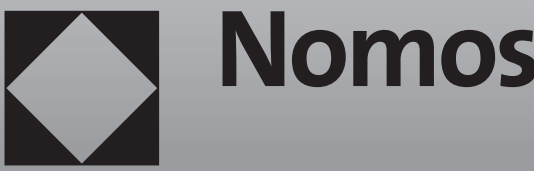

